\title{
MORFOECOLOGÍA TRÓFICA EN EL GÉNERO BARBUS (PISCES, CYPRINIDAE)
}

\author{
L. Encina \& C. Granado-Lorencio \\ Departamento de Ecología, Facultad de Biología, Universidad de Sevilla. 41080 Sevilla, España.
}

Palabras clave: Barbus, feeding, rnorphology, reservoirs, trophic adaptation.

\begin{abstract}
TROPHIC MORPHOECOLOGY IN THE GENUS BARBUS (PISCES, CYPRINIDAE)

In this paper the morphological features and feeding habits of three coexisting Barbus species in the Tajo basin (Spain) were studied. Fifteen biometric measurements of each specimen were taken, then eleven morphological variables were calculated, and at the same time, the gastric contents of each individual were analyzed.

The results showed and important relationship of barbillon length, body form, branchial filter and buccal apparatus to size, distribution and food items. The exploitation of available resources in the reservoirs is discussed in the light of the discriminant body differences of the species.
\end{abstract}

\section{INTRODUCCIÓN}

Las numerosas especies que integran la familia Cyprinidae se han adaptado a una amplia variedad de ambientes. La diversidad ecológica está directamente relacionada con la alta variabilidad morfológica de esta familia y, en concreto, sus muchas especializaciones tróficas ilustran la estrecha conexión entre la morfología de sus mecanismos de alimentación y su modo de vida (MATTHes, 1963). KeAst \& WebB (1966), WeRner (1977), Gatz (1979a, 1979b), MAtThews (1982), SchMitT \& Coyer (1983) y otros muchos autores, han demostrado la existencia de una estrecha relación entre ecología y morfología en los peces. Como fruto de estos trabajos se ha comprobado que numerosas adaptaciones morfológicas son el resultado evolutivo de procesos de competencia y posibilitan la coexistencia de especies y la minimización o anulación de dichos procesos. Estos fenómenos se acentúan entre especies congenéricas, ya que por su afinidad taxonómica y semejanza de vida se pueden suponer competidoras. En algunos

Limnetica, 6: 35-46 (1990)

(C) Asociación Española de Limnología, Madrid, Spain casos se ha comprobado que, cuando varias especies similares aparecen juntas, se manifiesta una tendencia hacia el desarrollo de ciertos caracteres y estructuras estrechamente relacionadas con la utilización de los recursos disponibles. A este fenómeno se le ha llamado divergencia de caracteres (MARGALEF, 1974).

El género Barbus posee la mayor diversidad específica de la fauna acuática española. Hasta la actualidad se han descrito nueve especies, ocho de ellas endémicas de la Península Ibérica (DOADRIO, 1984). Muchas de estas especies cohabitan en la misma cuenca hidrográfica; en concreto, en la del río Tajo coexisten Barbus bocagei, B. comiza, B. steindachneri y B. microcephalus (ALmaÇA, 1976; Collares-Pereira，1985; Granado-Lorencio \& ENCINA, 1988).

En el presente estudio se planteó la posible existencia de caracteres anatomo-funcionales divergentes relacionados con el régimen alimentício de tres de estas cuatro especies de barbos (B. microcephalus se excluyó, dado el escaso número de ejemplares capturados). Para ello se analizaron 11 caracteres morfológicos relacionados con la obtención del alimento, así como el contenido intestinal de cada una de las especies. 


\section{MATERIAL Y MÉTODOS}

\section{Muestreos}

Los peces fueron recolectados de los embalses de Torrejón y Valdecañas (río Tajo, Cáceres), en ocho campanas de muestreo realizadas entre febrero de 1984 y julio de 1985 . Se emplearon redes de tipo trasmallo de $12 \times 2 \mathrm{~m}$, situadas en 11 estaciones, 5 en el embalse de Torrejón y 6 en el de Valdecañas. Ambos embalses tienen características limnológicas de lago de zona templada holomíctico-monomíctico, con escasa mineralización y fuerte eutrofia de origen humano (MARGALEF et al., 1976). El estudio se basó en un total de 110 especímenes, 81 pertenecientes a B. bocagei, 19 a B. steindachneri y 10 a B. comiza. A fin de evitar diferencias en la alimentación debido a cambios ontogénicos, así como problemas de alometrías en el crecimiento de las especies para el análisis morfométrico, se seleccionaron únicamente ejemplares maduros y de tallas homogéneas (todos los especímenes pertenecen a las clases de edad $4+$ y $5+$ en el caso de B. bocagei y B. steindachneri; y a las clases de edad $5+$ y $6+$ los de B. comiza). Dado que no se registraron diferencias fisico-químicas ni biológicas, así como alimentarias en las distintas estaciones de muestreo, los ejemplares fueron agrupados y tratados en su totalidad.

\section{Biometría}

En el laboratorio, los ejemplares fueron identificados y separados por especies. A cada ejemplar se le tomaron 13 medidas biométricas (la longitud de los barbillones corresponde a la media del par derecho), con una precisión de $0,1 \mathrm{~mm}$ (figs. $1 \mathrm{y}$ 2). A partir de estas medidas se calcularon las variables de la tabla 1. La densidad de branquiespinas del primer arco branquia1 izquierdo se calculó midiendo su longitud y contando el número de branquiespinas.

\section{Alimentación}

Para la determinación de la dieta de las distintas especies se extrajeron los tractos intestinales de cada ejemplar y se analizó su contenido. Las presas se identificaron a nivel de especie, y se englobaron posteriormente en grupos más amplios según criterios de tamaño, hábitat o puramente taxonómicos. Se considero como detrito la materia orgánica no reconocible procedente del sedimento de los embalses. Para la evaluación de las dietas se siguió la metodología de GraNado-LoRENCIO y GarCía-Novo (1986). Se estableció una escala subjetiva de abundancia para cada uno de los componentes de la dieta (de 0 a 4 ) y se obtuvieron sus valores de frecuencia (proporción de estómagos con una determinada componente de la dieta respecto al total de estómagos). A partir de estos datos se aplicó el «índice de importancia en la alimentación» (IIA). Los resultados fueron

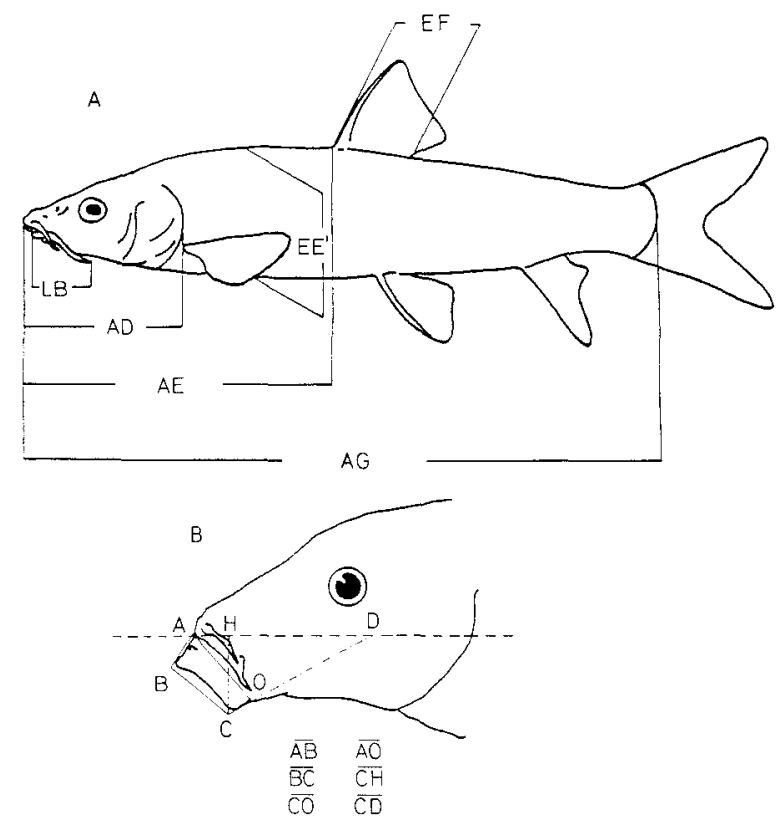

Figura 1.- Medidas biométricas efectuadas sobre el cuerpo. A) $\boldsymbol{A} \boldsymbol{G}$, longitud estandard; $\boldsymbol{A} \boldsymbol{E}$, longitud predorsal; $\boldsymbol{A D}$, longitud de la cabeza; $\boldsymbol{L B}$, longitud de los barbillones; $\boldsymbol{E} \boldsymbol{E}^{\prime}$, altura máxima del cuerpo; $E F$, longitud de la base de la aleta dorsal. B) $\boldsymbol{A} \boldsymbol{B}$, protrusión premaxilar; $\boldsymbol{B} \boldsymbol{C}$, amplitud de la apertura bucal; $\boldsymbol{C} \boldsymbol{O}$, longitud de la mandíbula inferior; $\boldsymbol{A} \boldsymbol{O}$, longitud de la base del embudo de succión; $\boldsymbol{C H}$, altura del labio inferior respecto al eje horizontal del cuerpo; $\boldsymbol{C D}$, longitud desde el labio inferior hasta el punto de corte entre el eje horizontal del cuerpo y la línea que une los labios superior e inferior.

Biometric measurements of the body. A) $\boldsymbol{A} \boldsymbol{G}$, standard length; $\boldsymbol{A} \boldsymbol{E}$, predorsal length; $\boldsymbol{A} \boldsymbol{D}$, head length; $\boldsymbol{L} \boldsymbol{B}$, barbels length; $\boldsymbol{E} \boldsymbol{E}^{\prime}$, maxim body height; $E F$, dorsal fin basis length. B) AB, premaxilar protrusion; $\boldsymbol{B} \boldsymbol{C}$, buccal opening amplitude; $\boldsymbol{C} \boldsymbol{O}$, lower jaw length; $\boldsymbol{A} \boldsymbol{O}$, suction funnel basis length; $\boldsymbol{C H}$, lower lip height in respect to horizontal body axis; $\boldsymbol{C D}$, length from lower lip to the point fo joining between the horizontal body axis and the upper-lower lip line. 

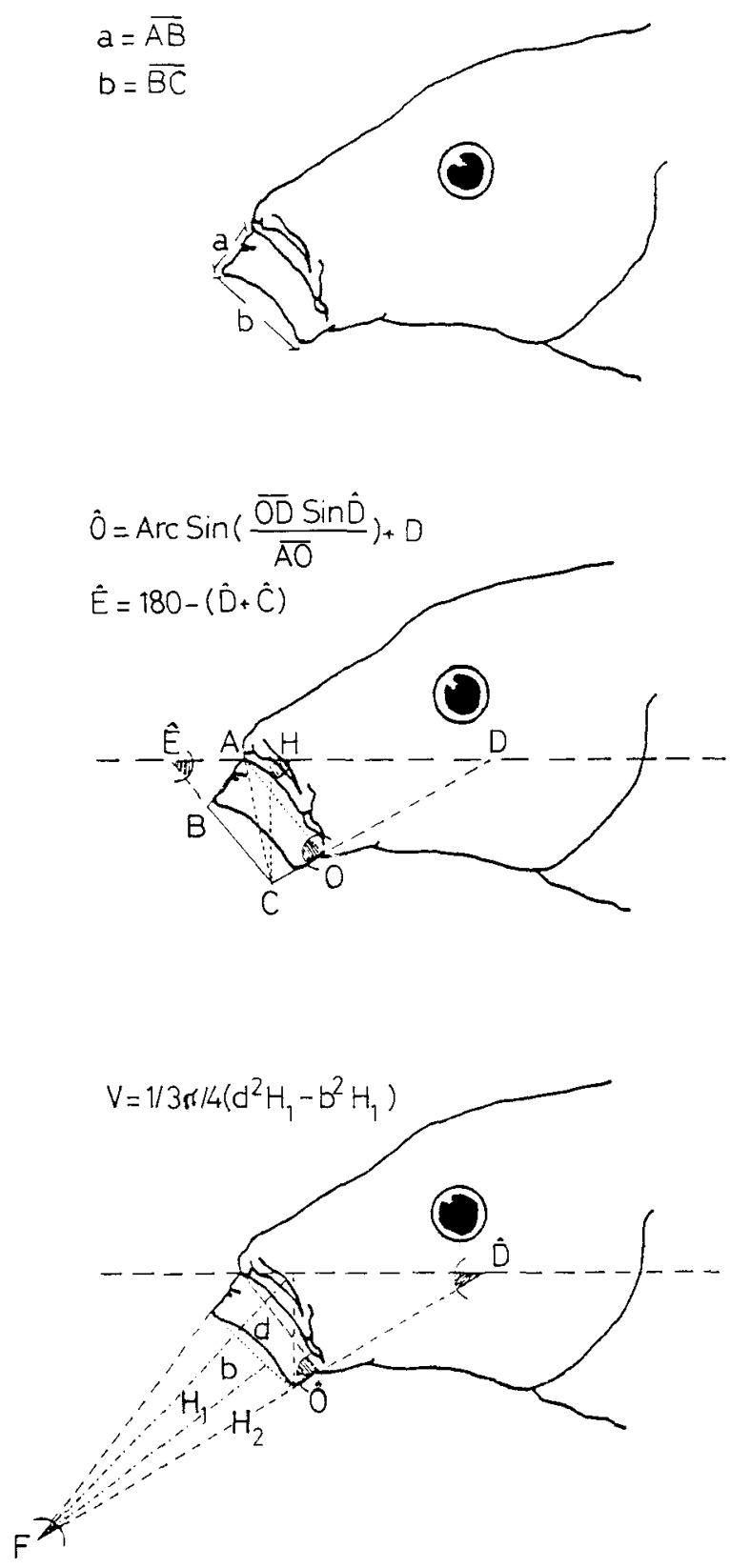

Figura 2.- Medidas biométricas de la boca (máxima protmsión y apertura bucal) de cada ejemplar y estima de los ángulos y volumen bucal. a, protmsión premaxilar; b, amplitud de la apertura bucal; $\hat{O}$, ángulo de giro de la mandíbula inferior; $\hat{E}$, ángulo de posición de la apertura bucal en relación al eje horizontal del cuerpo; V, volumen del embudo de succión. Mouth biometric measurements (maximum protrusion and buccal opening) of each specimen, along with angles and buccal volume estimation. a, premaxilar protmsion; b, buccal opening amplitude; $\hat{O}$, lower jaw rotation angle; $\hat{E}$, buccal opening position angle in respect to horizontal body axis; $\mathrm{V}$, suction funnel volume. agrupados (c.f. Guzlur, 1976) en «alimento principal» (IIA, mayor del $25 \%$ ), «alimento adicional» (IIA, entre el 15 y el $25 \%$ ) y «alimento esporádico (IIA, menor del $15 \%)$. Dado que interesaba relacionar caracteres morfológicoscon la alimentación general de las especies, se consideró el ciclo anual de la dieta, para lo cual se analizaron todos los tractos intestinales sin tener en cuenta la campaña en que fueron obtenidos. Pese a que existen diferencias estacionales en la alimentación de las especies que se estudian (ENCINA \& Granado-Lorencio, en prensa), el alimento principal de cada una de ellas se mantiene durante todo el ciclo anual, y coincide con los resultados del análisis global.

Para determinar posibles diferencias en las dietas de las especies respecto al tamaño de las presas, se midió la longitud de los elementos tróficos no alterados. En las medidas se excluyeron la longitud de la espina de la valva de los cladóceros y las espinas terminales que presentan algunos rotíferos (Eggers, 1982; Mc Commas \& Drenner, 1982; UNGER \& LEWIS, 1983).

\section{Análisis estadístico}

Para el estudio morfológico se calculó la media y la desviación típica de cada una de las variables estudiadas por especie. Para las comparaciones interespecíficas se aplicó el test de la t de Student (Sokal \& Rohlf, 1979). Para probar las posibles diferencias en el microhábitat alimenticio de las especies que se estudian respecto a la cantidad relativa de presas (IIA) consumidas, se emplearon los tests de Kruskall-Wallis y U de Mann-Whitney (Dixon, 1975).

\section{RESULTADOS}

\section{Morfología}

En la tabla 2 se muestran los valores de la media y desviación típica, así como los resultados de la t de Student para las variables morfológicas incluidas en el presente trabajo.

El índice cefálico obtiene un alto valor discriminante para las especies estudiadas; B. comiza posee la cabeza más larga, comprendida algo más 
Tabla 1.- Variables morfológicas (ver figuras 1 y 2). Morphological variables (see figures 1 and 2).

\begin{tabular}{|c|c|c|c|c|}
\hline Variables & Medidas & Significado & Sigla & Referencia \\
\hline fndice cefálico & $\begin{array}{l}\text { Longitud estándar dividida por la } \\
\text { longitud cefálica }\end{array}$ & $\begin{array}{l}\text { Número de veces que está com- } \\
\text { prendida la cabeza en la longitud } \\
\text { del pez }\end{array}$ & $\mathrm{AG} / \mathrm{AD}$ & ALMAÇA, 1965, 1972, 1981, 1982 \\
\hline \multirow[t]{2}{*}{ Índice de forma } & $\begin{array}{l}\text { Longitud estándar dividida por la } \\
\text { altura máxima del cuerpo }\end{array}$ & Forma del cuerpo del pez & $\mathrm{AG} / \mathrm{EE}$ & $\begin{array}{l}\text { ALMAÇA, 1972, 1976, } 1981 \\
\text { CASTELLO, } 1983 \\
\text { GRANADO-LORENCIO, } 1983 \\
\text { GUILLÉN, } 1982 \\
\text { ROJO \& RAMOS, } 1982\end{array}$ \\
\hline & $\begin{array}{l}\text { Longitud cefálica dividida por la } \\
\text { longitud de la base de la aleta } \\
\text { dorsal }\end{array}$ & $\begin{array}{l}\text { Asociado a caracteres hidrodiná- } \\
\text { micos del pez }\end{array}$ & ADIEF & ALMAÇA, 1976, 1981, 1982 \\
\hline \multirow[t]{2}{*}{ Razones } & $\begin{array}{l}\text { Longitud estándar dividida por la } \\
\text { longitud predorsal }\end{array}$ & Posición de la aleta dorsal & $\mathrm{AG} / \mathrm{AE}$ & ALMAÇA, 1976, 1981, 1983 \\
\hline & $\begin{array}{l}\text { Longitud de los barbillones divi- } \\
\text { dida por la longitud cefálica (me- } \\
\text { dia entre el anterior y posterior) }\end{array}$ & $\begin{array}{l}\text { Desarrollo de los barbillones bu- } \\
\text { cales }\end{array}$ & $\mathrm{LB} / \mathrm{AD}$ & $\begin{array}{l}\text { ALMAÇA, 1972, } 1976 \\
\text { CASTELLO, } 1983 \\
\text { GUILLEN, } 1982\end{array}$ \\
\hline $\begin{array}{l}\text { Densidad de branquies- } \\
\text { pinas }\end{array}$ & $\begin{array}{l}\text { Número de branquiespinas del } \\
\text { primer arco branquial dividido } \\
\text { por la longitud del arco }\end{array}$ & Eficiencia del filtro branquial & D. $\mathrm{Br}$ & LAGLER et al., 1977 \\
\hline Protrusibilidad & $\begin{array}{l}\text { Longitud desde el borde inferior } \\
\text { del morro al labio superior (con } \\
\text { máxima apertura bucal) dividida } \\
\text { por la longitud cefálica }\end{array}$ & $\begin{array}{l}\text { Protrusión relativa de la prema- } \\
\text { xila }\end{array}$ & $\mathrm{a} / \mathrm{AD}$ & $\begin{array}{l}\text { DE LA HOZ \& DYER, } 1984 \\
\text { GOSLINE, } 1981 \\
\text { LIEM, 1967, } 1978 \\
\text { PITCHER \& HART, } 1982 \\
\text { VAN HASSELT, } 1979\end{array}$ \\
\hline Amplitud bucal & $\begin{array}{l}\text { Longitud desde el labio superior } \\
\text { al inferior (con máxima apertura } \\
\text { bucal) dividida por la longitud ce- } \\
\text { fálica }\end{array}$ & $\begin{array}{l}\text { Diámetro de apertura relativa de } \\
\text { la boca }\end{array}$ & $\mathrm{b} / \mathrm{AD}$ & $\begin{array}{l}\text { ALEXANDER, 1969, } 1970 \\
\text { DE LA HOZ \& DYER, } 1984\end{array}$ \\
\hline Ángulo 6 & $\begin{array}{l}\text { Ángulo incluido entre la mandí- } \\
\text { bula infenor y la base del embu- } \\
\text { do de succión }\end{array}$ & $\begin{array}{l}\text { Máxima rotación de la mandíbu- } \\
\text { la inferior sobre la unión artícu- } \\
\text { lo-cuadrado }\end{array}$ & $\hat{O}$ & Presente trabajo \\
\hline Ángulo E & $\begin{array}{l}\text { Ángulo incluido entre el eje hori- } \\
\text { zontal del cuerpo y la proyección } \\
\text { de la línea imaginaria que une los } \\
\text { labios superior e inferior }\end{array}$ & $\begin{array}{l}\text { Posición de la apertura bucal en } \\
\text { relación al cuerpo }\end{array}$ & $\hat{\mathrm{E}}$ & Presente trabajo \\
\hline Volumen bucal & $\begin{array}{l}\text { Volumen de un tronco de cono } \\
\text { oblicuo dividido por la longitud } \\
\text { cefálica }\end{array}$ & $\begin{array}{l}\text { Volumen relativo del embudo de } \\
\text { succión }\end{array}$ & V/AD & $\begin{array}{l}\text { MULLER } \text { et al., } 1982 \\
\text { MULLER \& OSSE, } 1984 \\
\text { VAN LEEWEN \& MULLER, } 1984\end{array}$ \\
\hline
\end{tabular}

de tres veces en su longitud standard; en el extremo opuesto se halla B. bocagei, con la cabeza comprendida más de cuatro veces en la longitud standard; B. steindachneri ocupa una situación intermedia. Las razones AG/AE y ADIEF (fig. 1) permiten igualmente una buena discriminación de las especies; B. comiza tiene la aleta dorsal retrasada respecto a B. bocagei, y B. steindachneri posee valores intermedios. B. comiza consigue el valor más alto para el índice $\mathrm{AD} / \mathrm{EF}$, al poseer una longitud de la cabeza mayor, junto con la menor longitud de la base de la aleta dorsal, y es opues- to a B. bocagei. El índice de forma es mayor en B. comiza (cuerpo más fusiforme e hidrodinámico). En cuanto a la longitud de los barbillones, el mayor desarrollo lo presenta B. bocagei (especialmente de los barbillones traseros). La densidad de branquiespinas es distinta para las tres especies, siendo la de B. bocagei la mayor (tabla 2).

Respecto a la morfología bucal, B. comiza difiere de las otras dos especies por el valor del ángulo $\mathbf{E}$ (tabla 2); su valor más alto se traduce en que la posición de la abertura bucal es la más adelantada en-dirección dorsal. Por el contrario, el 
Tabla 2.- Media $(\overline{\mathrm{X}})$, desviación estandard (SD) e intervalos de confianza del test de la t de Student (++99\%; +95\%; - no significativo) (B.b = Barbus bocagei; B.s = B. steindachneri; B.c = B. comiza).

Mean $(\bar{X})$, standard desviation (SD) and confidence limits of the Student-t test $(++99 \% ;+95 \% ;-$ no significant $)(B . b=B a r b u s$ bocagei; B.s = B. steindachneri; B.c = B. comiza).

\begin{tabular}{|c|c|c|c|c|c|c|c|c|c|}
\hline \multirow[b]{2}{*}{ Variables } & \multicolumn{2}{|c|}{$\begin{array}{l}\text { Barbus } \\
\text { bocagei }\end{array}$} & \multicolumn{2}{|c|}{$\begin{array}{c}\text { Barbus } \\
\text { steindachneri }\end{array}$} & \multicolumn{2}{|c|}{$\begin{array}{l}\text { Barbus } \\
\text { comiza }\end{array}$} & \multicolumn{3}{|c|}{ Intervalos de confianza } \\
\hline & $X$ & $S D$ & $X$ & $S D$ & $x$ & $S D$ & B.b.-B.s. & B.b.-B.c. & B.s.-B.c. \\
\hline $\mathrm{AG} / \mathrm{AD}$ & 4,122 & 0,138 & 3,860 & 0,131 & 3,311 & 0,162 & ++ & ++ & ++ \\
\hline AGIEE' & 5,007 & 0,363 & 5,050 & 0,341 & 5,387 & 0,311 & - & ++ & ++ \\
\hline $\mathrm{AD} / \mathrm{EF}$ & 2,018 & 0,140 & 2,207 & 0,134 & 2,588 & 0,240 & ++ & - & ++ \\
\hline $\mathrm{AG} / \mathrm{AE}$ & 2,039 & 0,050 & 1,994 & 0,030 & 1,916 & 0,046 & ++ & ++ & ++ \\
\hline LB/AD & 0,245 & 0,028 & 0,210 & 0,021 & 0,147 & 0,226 & ++ & ++ & $+t$ \\
\hline D. $\mathrm{Br}$ & 0,806 & 0,209 & 0,676 & 0,059 & 0,515 & 0,124 & + & ++ & ++ \\
\hline $\mathrm{a} / \mathrm{AD}$ & 0,150 & 0,030 & 0,145 & 0,030 & 0,090 & 0,030 & - & ++ & ++ \\
\hline $\mathrm{b} / \mathrm{AD}$ & 0,254 & 0,030 & 0,244 & 0,020 & 0,243 & 0,030 & - & - & - \\
\hline 6 & 63,250 & 6,910 & 59,580 & 3,870 & 49.310 & 3,920 & + & ++ & ++ \\
\hline$\hat{\mathbf{E}}$ & 48,130 & 6,870 & 48,770 & 6,590 & 63,200 & 8,240 & - & ++ & $+t$ \\
\hline V/AD & 84,250 & 34,750 & 97,840 & 18,590 & 209,530 & 96,210 & $-m$ & $+t$ & ++ \\
\hline
\end{tabular}
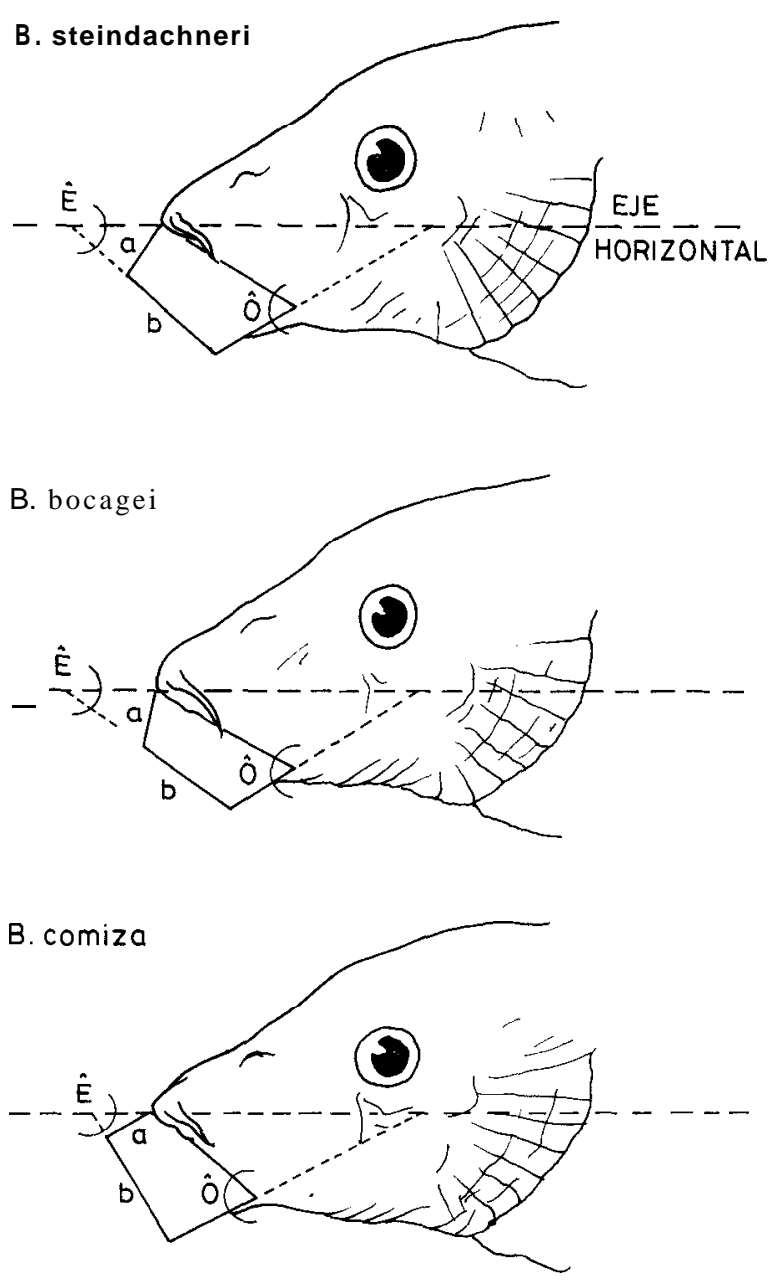

ángulo de giro de su mandíbula inferior es menor, lo mismo que su protrusión premaxilar, siendo la dirección de ésta levemente oblicua en posición ventral. La localización del perfil bucal de B. comiza es así el más terminal de las especies estudiadas (fig. 3). El volumen del embudo de succión es mayor en esta especie.

B. bocagei se caracteriza por poseer la mayor protrusibilidad bucal, y la dirección de ésta es la más ventral respecto al eje horizontal del cuerpo. Registra también el mayor valor para el ángulo 0 $\mathrm{y}$, por el contrario, el menor volumen del embudo de succión.

B. steindachneri presenta claras diferencias con B. comiza, y menos con B. bocagei. Su protrusibilidad es intermedia y dirigida ventralmente, aunque en menor grado que en B. bocagei, con lo que resulta un valor del ángulo $\mathrm{E}$ más cercano al de B. comiza. Igualmente registra valores intermedios para el ángulo 0 y el volumen del embudo de succión.

Figura 3.- Modelo del perfil bucal para cada especie. a, protrusión premaxilar; b, amplitud de la apertura bucal; $O$, ángulo de giro de la mandíbula inferior; $\hat{E}$, ángulo de posición de la apertura bucal en relación al eje horizontal del cuerpo. Buccal profile model of each fish species. a, premaxilar protrusion; b, buccal opening amplitude; $\mathrm{O}$, lower jaw rotation angle; $\hat{E}$, buccal opening position angle in respect to horizontal body axis. 


\section{Barbus bocagei Barbus steindachneri Barbus comiza}

Rotíferos

Cladóceros

Huevos de cladóceros

Efipios

Ostrácodos

Ciclópidos

Diaptómidos

Larvas de quironómidos

Larvas de tricópteros

Insectos

Restos macroinvertebrados

Puestas de peces

Escamas

Peces

Restos de peces

Semillas

Restos vegetales

Arena

Detritos

Cianofíceas

Diatomeas

Clorofíceas
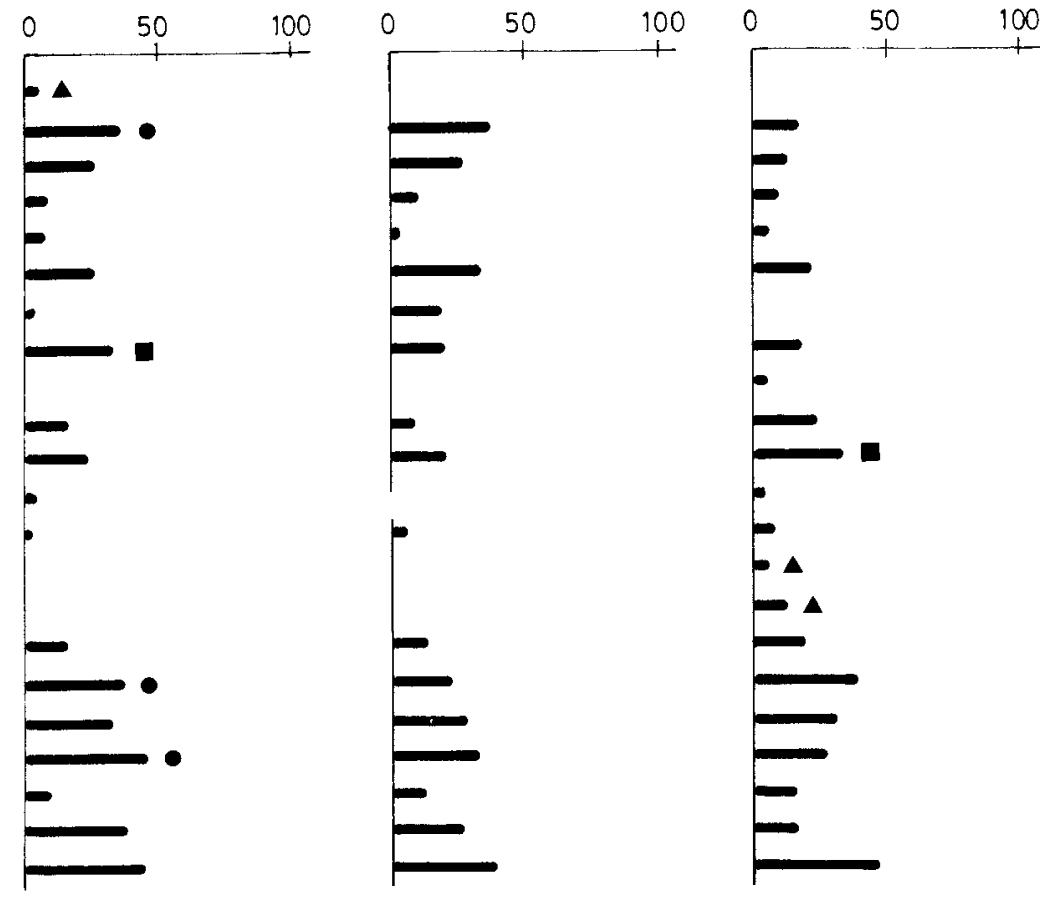

Bosmina

Leydigia

Alona

Chydorus

Daphnia hyalina

D. pulex

Ceriodaphnia

Daphnia magna

Iliocryptus

Dípteros

Formícidos

Coleópteros

Micronecta

Cianofíceas coloniales

Cianofíceas filamentosas

Diatomeas filamentosas

Diatomeas centrales

Diatomeas pinnadas

Clorofíceas filamentosas
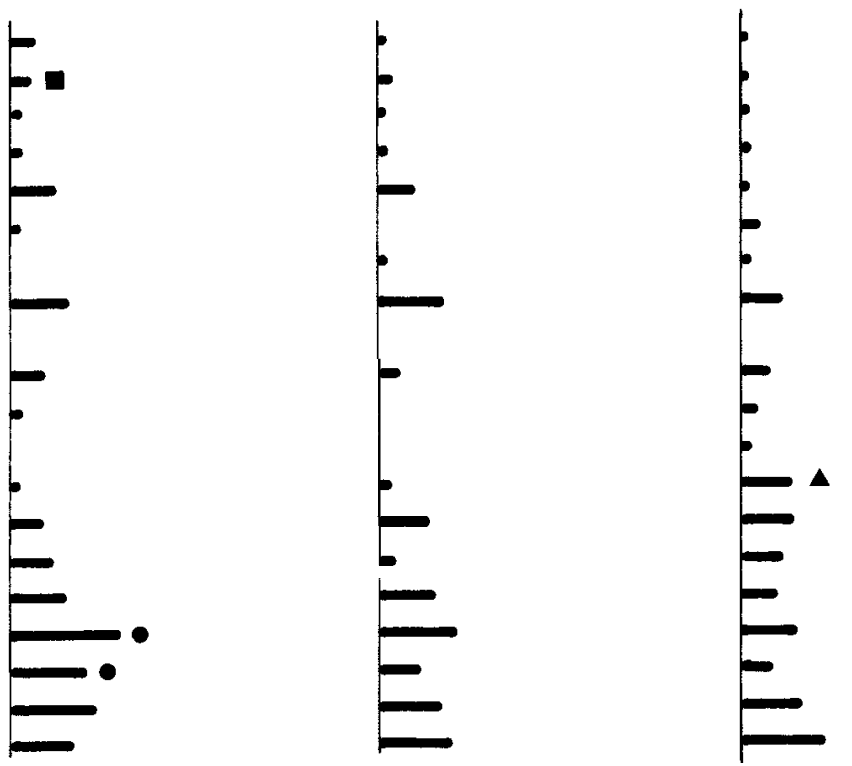

$\triangle \mathbf{P}=0,01$

- $P=0,05$

- $\mathrm{P}=0,1$

Figura 4.- Porcentaje del f́ndice de Importancia Alimenticia y resultados del test de Kruskall-Wallis y U de Hann-Withney (se representan sólo los componentes tróficos con significación estadística).

Precentages of the Feeding Importance Index and results of Kruskall-Wallis and Mann-Withney U test (only the food items with statistical differences were represented). 


\section{Alimentación}

La alimentación de $B$. bocagei está dominada por larvas de quironómidos, detrito (al que se asocia arena), cladóceros, restos vegetales y algas, fundamentalmente diatomeas centrales (típicamente Cyclotella). El alimento adicional lo constituyen puestas de cladóceros, ciclópidos, restos de macroinvertebrados acuáticos y otras algas planctónicas. El alimento esporádico lo forman presas o estructuras de aparición temporal, tanto del plancton, como insectos terrestres (dípteros, formícidos, etc.), materiales sedimentarios y flotantes (escamas de peces, semillas...) (fig. 4).

El régimen trófico de $B$. steindachneri está constituido por crustáceos planctónicos (cladóceros y ciclópidos), acompañados de detrito y clorofíceas. Las larvas de quironómido aparecen como alimento adicional, junto con huevos de cladóceros, restos de macroinvertebrados acuáticos y las restantes algas. El alimento esporádico lo constituyen efipios, ostrácodos, semillas y pupas de quironómidos, entre otros.

El alimento principal en $B$. comiza lo forman restos de macroinvertebrados acuáticos, restos vegetales y clorofíceas. Como alimento adicional entran a formar parte ciclópidos, larvas de quironómido, insectos (especialmente Micronecta), semillas, detrito y otras algas planctónicas. Entre el alimento esporádico, destacan los peces y restos de peces; ésta es la única especie de las estudiadas que presenta rasgos de ictiofagia en su dieta.

El alimento principal no sufre estacionalmente cambios sustanciales en las tres especies; los cambios afectan sólo a los elementos de la dieta que componen el alimento adicional y esporádico (ENcina \& Granado-Lorencio, en prensa). Los resultados de los tests de Kruskall-Wallis y MannWhitney muestran la existencia de diferencias significativas $(\mathrm{P}<0,1)$ en la importancia de algunos componentes alimenticios de la dieta de estas tres especies de barbos (fig. 4). Así, en B. bocagei la presencia de quironómidos, detrito, restos vegetales y diatomeas no centrales es significativamente mayor que en $B$. steindachneri. B. bocagei se diferencia de B. comiza en un mayor consumo de cladóceros (especialmente cladóceros pequeños), larvas de quironómidos, detrito y diatomeas. Por el contrario, la presencia de insectos acuáticos (Micronecta), restos de macroinvertebrados acuá- ticos, peces y cianofíceas coloniales es significativamente mayor en la dieta de B. comiza. Este mayor consumo de macroinvertebrados y peces diferencia también la dieta de $B$. comiza de la de B. steindachneri.

B. bocagei destaca del resto de las especies por la mayor participación en su dieta de presas de talla pequeña: rotíferos (no consumidos por ninguna de las otras dos especies: talla media 0,173 $\mathrm{mm})$; los cladóceros Bosmina (0,366 mm), Chydorus $(0,454 \mathrm{~mm})$, Leydigia $(0,860 \mathrm{~mm})$, Alona $(0,713)$ e Iliocryptus $(0,907)$; y ostrácodos $(0,589$ $\mathrm{mm}$ ). Todos ellos corresponden a las categorías alimenticias más pequeñas de las encontradas en todos los tractos digestivos analizados. B. steindachneri tiene también una importante participación en su alimentación de cladóceros de talla pequeña, pero destaca la ausencia de rotíferos. La presencia de elementos de talla pequeña en los contenidos intestinales de B. comiza es escasa, mientras que la ocurrencia de cladóceros de gran talla como Daphnia magna $(2,038 \mathrm{~mm})$ o insectos (1,770 de talla media para el género Micronecta, 3,900 $\mathrm{mm}$ para formícidos o $6,500 \mathrm{~mm}$ para dípteros) es más importante. B. comiza es la especie en la que se ha registrado el mayor tamaño de presa (peces). Encina \& Granado-LoRencio (en prensa) encuentran que estas diferencias se registran igualmente cuando se analiza la alimentación estacionalmente.

\section{DISCUSIÓN}

Los peces, al igual que otros organismos, presentan regímenes alimenticios distintos, de acuerdo con la estructura y morfología de los órganos que participan en la detección y captura del alimento, la biología propia de las especies y la disponibilidad de recursos en el medio. Numerosos estudios han discutido el papel que juegan las diferencias morfológicas en la composición cualitativa de la alimentación de distintas especies coexistentes, ya sea correlacionadas con divergencias en el hábitat alimenticio (Werner \& Hall, 1977; Magnan \& Fitzgerald, 1982; Van OiJen, 1982), con la capacidad de selección de las presas (NILSsOn, 1979; GREENFIELD et al., 1983; LAUR \& EBELING, 1983; WALSH \& FitzGERALD, 1984) o más fre- 
cuentemente con ambos, resaltando la importancia que tienen estas interacciones en la organización de la comunidad (HEsPENHEIDE, 1973). GATZ (1979a) llega incluso a concluir que cualquier característica morfológica que aparezca con diferencia significativa entre dos especies, puede asumirse para indicar patrones óptimos de utilización de recursos. En este sentido, y asumiendo las limitaciones que conlleva este planteamiento determinista, pueden ser inferidos desde las significaciones estadísticas, comportamientos diferenciales de tipo ecológico (papel en el ecosistema). Estas diferencias están referidas esencialmente a caracteres morfológicos y merísticos, y a la estructura del aparato mandibular (RINGLER, 1979; WERNER, 1979), y hacen referencia, en definitiva, a un concepto tan largamente desarrollado como controvertido: el nicho trófico (GATZ, 1979a, 1981; Gladfelter \& Johnson, 1983). Según Schoener (1974) los tres componentes principales que definen el nicho trófico de una especie son: el tipo de presa, el tamaño y la localización del alimento.

En relación con la localización espacial, la alimentación de cada una de las especies de barbo estudiadas parece reflejar tendencias específicas de explotación preferencial en el ecosistema. Estas tendencias son potenciadas por determinadas características morfológicas (como el desarrollo de los barbillones, la posición de la boca y la dirección de protrusión bucal) que aportan una mayor ventaja o aptitud para alimentarse en los distintos hábitats de la masa de agua.

La mayoría de los autores coinciden en señalar que los barbillones bucales tienen un mayor desarrollo en las especies que se alimentan del bentos (Keast \& WebB, 1966; Margalef, 1983). B. bocagei tiene un mayor desarrollo de los barbillones que las otras especies, lo que parece estar de acuerdo con los datos obtenidos acerca de su composición alimenticia, según la cual esta especie tendría hábitos preferentemente bentófagos. En los contenidos analizados son importantes las larvas de quironómido (MOYLe, 1973; Werner \& Hall, 1977; Nilsson, 1979; Stenson, 1979), la arena (STICKNEY, 1976) y grandes diatomeas (GATZ, 1981). Este gran desarrollo de sus barbillas (que actúan como órganos quimiotactiles e intervienen en la detección de las presas; RINGLER, 1979) le posibilitaría una más fácil localización de los organismos del bentos, alimento principal durante todo el ciclo anual. La presencia de barbillones en B. steindachneri y B. comiza también posibilita una alimentación asociada al bentos; sin embargo la eficiencia en la detección de presas es probablemente inferior a la de la anterior especie, debido a que están menos desarrollados (principal-

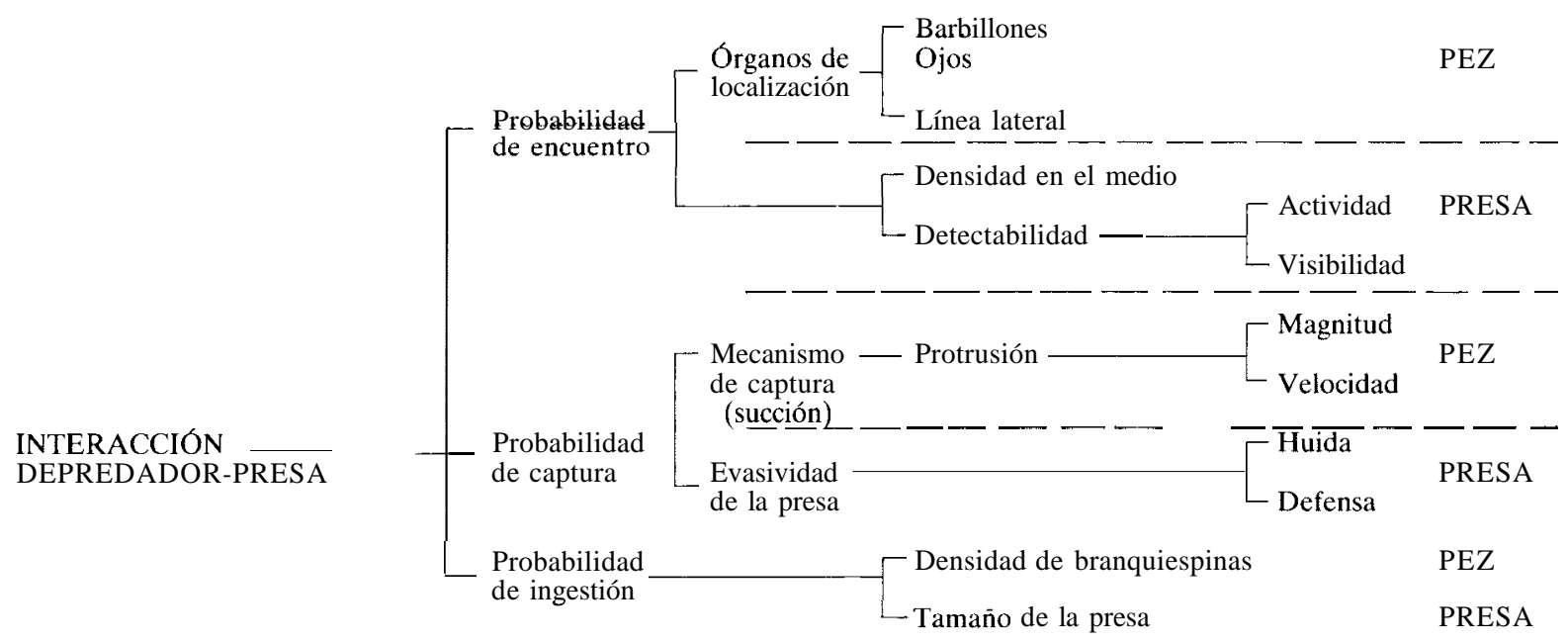

Figura 5.- Modelo de interacción predador-presa y factores que intervienen Model of predator-prey interactions and affecting factors. 
mente en B. comiza). De hecho, ambas especies han registrado una alimentación de características más limnéticas, con mayor importancia de crustáceos planctónicos.

El índice de forma es otra de las variables biométricas que ha sido tradicionalmente asociada al hábitat de los peces (MATTHES, 1963), y recientemente, a las ventajas relacionadas con el proceso de captura de presas evasivas (VAN OIJEN, 1982). Un cuerpo más fusiforme facilita la natación. Así, un mayor valor del índice de forma correspondería a especies más veloces, con posibilidad de capturar presas más huidizas (WERNER, 1977; MATTHEws, 1982). El índice de forma de B. comiza es el mayor de los registrados, además la cabeza está considerablemente desarrollada en la región preorbital (morro), en sentido longitudinal, lo que le confiere unas excepcionales características hidrodinámica Por otra parte, la posición más retrasada de la aleta dorsal es propia de especies veloces y adaptadas a una estrategia alimenticia de acecho y captura de presas muy evasivas, como por ejemplo peces. B. comiza seria una especie con posibilidades para una alimentación ictiófaga, como lo corroboran los resultados.

La magnitud de la protrusión es también un elemento importante. MATthes (1963) señala que una mayor protrusión es típica de peces ligados a una alimentación más bentónica, mientras que una protrusión pequeña corresponde a organismos de aguas libres. Los resultados obtenidos en este trabajo parecen apoyar esta teoría (B. bocagei registra la mayor protrusibilidad y B. comiza la menor).

No sólo resulta de gran relevancia la amplitud de la apertura y protrusión bucal, sinó también la dirección de ésta respecto al eje horizontal del cuerpo. En numerosos trabajos se ha comprobado que la posición de la boca y la protrusión están relacionadas con el hábitat explotado por las distintas especies de peces y, por lo tanto, con el tipo de presas consumido (AlEXANDER, 1966, 1967; Ringler, 1979; GATZ, 1981; AdAMICKA, 1983). Una boca protrusible en dirección ventroterminal indica alimentación de fondo, con una dieta caracterizada esencialmente por detrito orgánico y larvas de quironómido, pudiendo acompañarse adicionalmente de cladóceros y copépodos de la columna de agua (KEAst \& WebB, 1966). Este es el caso de B. bocagei. Por el contrario, una boca protrusible en dirección dorso-terminal o terminal está ligada a especies que se alimentan de la columna de agua y en superficie (típicamente insectos, semillas flotantes y pupas de quironómidos) (KEAsT \& WEBB, ops. cit.); B. comiza encajaría en este segundo grupo. B. steindachneri sería una forma intermedia, con posibilidades de explotar indiferentemente ambos subsistemas; su perfil, más parecido al de B. comiza, estaría más adaptado a una explotación limnética, y menos eficiente para la alimentación ligada al sedimento que $B$. bocagei.

El límite inferior de tamaño de presa ingerible por un pez viene dado por la constitución del filtro branquial (referida a los espacios interbranquiespínicos) (Uribe-Zamora, 1975; SCHMitT \& COYER, 1983). Según esto el consumo de cantidades importantes de algas coloniales y filamentosas puede ser atribuible a la constitución del filtro branquial (DRENNER et al., 1984). Los valores medios registrados para la densidad de branquiespinas en B. bocagei, B. steindachneri y B. comiza fueron, respectivamente, 0,806, 0,676 y 0,515 branquiespinas $/ \mathrm{mm}^{2}$. Estos resultados parecen ser los responsables de las diferencias de tamaño de presa (fundamentalmente del plancton) encontradas en estas tres especies. El límite superior de tamaño de presa viene determinado por la amplitud de la abertura bucal y su volumen (UNGER \& LEwIs, 1983). Dado el rango de tamaño ingerido por estas especies de barbos, no parecen ser estas variables las determinantes de posibles diferencias tróficas entre ellas, salvo quizá para B. comiza, que incluye peces en su alimentación; la ingestión de este tipo de presas sí está claramente limitada por la amplitud bucal. Sin embargo, el valor medio registrado para esta variable es similar en las tres especies; es el volumen bucal el que resulta $\left(111,69 \mathrm{~mm}^{3}\right)$ más que en la especie inmediatamente inferior) superior. Teniendo en cuenta que el mecanismo principal de alimentación en el barbo es la succión, y que ésta depende del volumen bucal (AlEXANDER, 1966), parece lógico pensar que la presión de succión necesaria para la captura de peces ha de ser superior y requiere un mayor volumen bucal.

En el tipo de presa consumido participan, no sólo las características anatómicas del pez, sinó también las de la propia presa, lo cual es consecuencia directa de una interacción depredador- 
presa (coevolución). Según Gerritsen \& StrickLER (1977) esta relación puede ser dividida en una secuencia de varios sucesos independientes: encuentro, ataque, captura e ingestión. Como se observa en la figura 5, la mayoría de las especializaciones morfológicas referidas para cada especie de barbo determinarán, en parte, el tipo de alimento consumido por ellas. Atendiendo al tipo de alimento consumido y a ciertas características morfológicas, MatThes (1963) elaboró una clasificación ecomorfológica para los barbos africanos. Según ésta, B. comiza quedaría incluido dentro de las formas que dicho autor denomina de aguas libres. Las especies de este grupo tienen típicamente una boca alargada, terminal y poco protráctil, el cuerpo es fusiforme, son generalmente carnívoros, y se alimentan de insectos, crustáceos zooplanctónicos y peces.

Las especies de vida bentónica, como B. bocagei, se caracterizan por un gran desarrollo de los barbillones bucales, tienen menor valor del índice de forma y la boca es ínfera con protrusión ventral. Son principalmente omnívoros o detritívoros. Sus presas típicas son lamas de quironómido, aunque ingieren también organismos del plancton. En estos peces detritívoros es típica la elevada presencia de algas en su alimentación, ya que éstas suelen tapizar las superficies duras del sustrato y son fácilmente desprendidas por la posición ínfera de la boca y la ayuda del labio superior protráctil. También son consumidas por succión aquellas algas que se acumulan en los fondos por sedimentación (DE SILVA et al., 1980; DrenNER et al., 1984); ello explicaría la abundancia de diatomeas grandes, como Cyclotella, Melosira o Skeletonema, en los contenidos intestinales de esta especie.

Las características morfológicas y alimentarias de B. steindachneri no se ajustan estrictamente a ninguno de los dos ecomorfos anteriores; esta especie posee rasgos intermedios que le posibilitan tanto una forma de vida bentónica como de aguas libres, aunque posiblemente con menor eficiencia que las anteriores especies.

No obstante, la clasificación propuesta por MatThes (1963) resulta hasta cierto punto teórica e idealista; en la práctica, la distinción entre especies ícticas limnéticas, planctófagas y bentónicas es bastante imprecisa en las aguas epicontinentales (MARGALEF, 1983), especialmente en los lagos y embalses que, como los de Torrejón y Valdecañas, se localizan en climas templados. En ellos, el ciclo limnológico de la masa de agua da lugar a cambios temporales de la disponibilidad de los recursos en el eje plancton-bentos, en detrimento de una clara especialización trófica de la comunidad íctica. Estos cambios son la causa de que la alimentación de estas especies presente un amplio rango de tipos de presas consumidas. Esta plasticidad trófica es un resultado concordante con lo que la teoría predice en el caso de especies que habitan en ecosistemas cambiantes; éstas han de adaptarse a los cambios de recursos con cambios en sus preferencias alimentarias (ANGERMEIER, 1982).

La escasa presencia de ecosistemas acuáticos lénticos en la Península Ibérica determinó que las adaptaciones morfológicas desarrolladas por el grupo taxonómico Barbus se realizaran de acuerdo con las características funcionales del ecosistema lótico, hábitat preferentemente ocupado por estas especies. Así pues, las relaciones ecomorfológicas encontradas en este trabajo y sus relaciones con la dinámica funcional de los embalses (ciclo anual, pulsos de productividad, espectro de alimentación, etc.), tienen que ver más con capacidades explotadoras de los recursos, desarrolladas con anterioridad a la colonización del nuevo ecosistema, que con procesos de tipo determinista surgidos por la interacción competitiva (coexistencia) de la comunidad íctica actual. Es a partir del establecimiento de una nueva asociación de especies en el embalse cuando se produce un proceso de exclusión de aquellas menos adaptadas a las nuevas condiciones, y la divergencia de caracteres puede considerarse como el resultado final de procesos interactivos (principio de exclusión competitiva).

Puede concluirse que las especies B. bocagei, B. steindachneri y B. comiza presentan unas particulares características morfológicas que les permiten hacer un uso diferencial de los recursos tróficos cuando la disponibilidad de éstos en el medio es favorable, pero que al mismo tiempo les posibilita un alto grado de plasticidad en su alimentación acorde con los cambios limnológicos de la masa de agua en que viven. 
Bibliografía

ADAMicKA, P., 1983. Contributions to the functional anatomy of feeding apparatus of five cyprinids of Parakrama Samudra (Sri-Lanka). In: Schiener, F. (ed), Limnology of Parakrama Samudra - Sri Lanka, 171-175. The Hague.

Alexander, R. McN., 1966. The functions and mechanisms of the protrusible upper jaws of two species of cyprinid fish. J. Zool 149: 288-296. London.

AleXANDER, R. McN., 1967. The functions and mechanisms of the protrusible upper jaws of some acanthopterygian fish. J. Zool 151: 43-64. London.

AleXANDER, R. McN, , 1969. Mechanics of the feeding action of a cyprinid fish. J. Zool 159: 1-15. London.

AleXANDER, R. McN., 1970. Mechanics of the feeding action of varius teleost fishes. J. Zool 162: 145-156. London.

AlmaçA, C., 1965. Contribution a la connaissance des poissons des eaux intérieures du Portugal. Arq. Mus. Boc. (2. ser.) 1 (2): 9-39.

AlmaÇA, C., 1972. Sur la systématique des barbeaux (genre et sousgenre Barbus) de la Penínsule Ibérique et de l'Afrique du nord. Arq. Mus. Boc. (2. ${ }^{\mathrm{a}}$ ser.) 3 (10): 319-346.

AlmaçA, C., 1976. Zoogeografia e especiaçao dos cipnnideos da Peninsula Ibérica. Bol. Soc. Port. cienc. Nat. 4: 1-28.

AlmaÇA, C., 1981. La collection de Barbus d'Europe du Muséum National d'Histoire Naturelle (Cyprinidae, Pisces). Bull. Mus. Natn. Hk. Nat. Paris, $4 .^{a}$ ser., 3 (sec. A, N. ${ }^{\circ}$ ): 277-307.

AlmaÇA, C., 1982. Re-examination of the types of Barbus haasi Mertens, 1924 (Pisces: Cyprinidae). Senckenbergiana biol. 63: 33-38.

AlmaçA, C., 1983. Notes on Barbus graellsii Steindachner, 1866. Ann. Naturhist. Mus. Wien. 85 (B): 1-7.

ANGermeIER, P.L., 1982. Resource seasonality and fish diets in an Illinois stream. Env. Biol. Fish. 7 (3): 251-264.

Castelló, V.J., 1983. Utilización de técnicas multivariantes en el estudio de la variabilidad morfológica de una población de barbos (Barbus barbus sclateri G.) (Pisces, Cyprinidae) en el sur de España. His. Nat. 3 (1): 1-15.

Collares-Pereira, M.J., 1985. Ciprinideos do Alentejo. Act. Congreso sobre o Alentejo (Evora) 2: 537-545.

DE LA Hoz, E. \& B. DYER, 1984. Mecanismo de protrusión premaxilar en Helicolenus lengerichi (Pisces, Scorpaenidae). Invest. Mar. Valparaiso 12: 27-50.

De Silva, S.S., P.R.T. Camaranatunga \& C.D. De Silva, 1980. Food, feeding ecology and morphological features associated with feeding of four co-occurring cyprinids (Pisces: Cyprinidae). Neth. J. Zool. 30 (1): 54-73.

DiXON, W.J., 1975. BMDP. Biomedical Computers Programs. University of California Press. USA. pp. 791.

DOADRIO, I., 1984. Relaciones filogenéticas y biogeográficas de los barbos (Barbus; Cyprinidae) de la Península Ibérica y aportes corológicos y biogeográficos a su ictiofauna continental. Tesis Doctoral. Universidad Complutense de Madrid.

Drenner, R. W., J.R. Mummert, F. Denoyelles \& D. KetTLE, 1984. Selective particle ingestion by a filter-feeding fish and its impact on phytoplankton community structure. Limnol. Oceanogr. 29 (5): 941-948.

DuTTA, H.M., 1979. Form and function of jaws during feeding: Ctenopoma acutirostre, Anabas testudinens and Macropodus opercularri. Acta Morphol. Neerl-Scand. 17: 119-132.
EGGERS, D.M., 1982. Planktivore preference by prey size. Ecology 63 (2): 381-390.

EnCinA, L. \& C. GRANAdo-Lorencio, en prensa. Trophic segregation and resource partitioning in three co-existing $\boldsymbol{B a r}$ bus species. Hydrobiologia.

GATZ, A.J., 1979a. Community organization in fishes as indicated by morphological features. Ecology 60: 711-718.

Gatz, A.J., 1979b. Ecological morphology of freshwater stream fishes. Tulane Stud. Zool. Bot. 21: 91-124.

Gatz. A.J., 1981. Morphologically inferred niche differentiation in stream fishes. Amer. Midl. Nat. 106 (1): 10-20.

Gerritsen, J. \& J.R. STRICKLER, 1977. Encounter probabilities and community structure in zooplankton: a mathematical model, J. Fish. Res. Bd. Can. 34: 73-82.

GladFelter, W.B. \& W.S. Johnson, 1983. Feeding niche in a guild of tropical reef fishes (Holocentridae). Ecology 64 (3): $552-563$.

GosLine, W.A., 1981. The evolution of the premaxillary protrusion system in some teleostean fish groups. J. Zool. 193: 11-23. London.

GranAdo-Lorencio, C., 1983. Ecología de la comunidad íctica del embalse de Arrocampo (cuenca del Río Tajo, Cáceres). Tesis Doctoral. Universidad de Sevilla. 364 pp.

Granado-Lorencio, C. \& F. Garcta-Novo, 1986. Feeding habits of the fish community in a eutrophic reservoir in Spain. Ekol. Pol. 34 (1): 95-110.

GranAdo-Lorencio, C. \& L. Encina, 1988. Nueva cita de Barbus microcephalus Almaça (Pisces, Cyprinidae) en España. Doñana Acta Vertebrata 15 (1).

GREENFIELD, D.W., C.F. RAKocinski \& T.A. GREENFIELD, 1983. Spatial interactions in wet and dry seasons between Gambusia luma and Gambusia sexradiata (Pisces: Poeciliidae) in Belize, Central America. Fieldiana. Zool. 14: 1-16.

Gullén, E., 1982. Estudio ecológico de la ictiofauna del embalse de Torrejón (Río Tajo, Parque Natural del Monfragüe, Cáceres). Tesis de Licenciatura. Universidad de Sevilla. 199 pp.

GuzIUR, J., 1976. The feeding of two years old carp (Cyprinus carpio L.) in a vendance lake Klawoj. Ekol. Pol. 24 (2): 211-235.

HESPENHEIDE, H.A., 1973. Ecological inferences from morphological data. Ann. Rev. Ecol. Syst. 4: 213-229.

KEAST, A. \& D. WEBB, 1966. Mouth and body form relative to feeding ecology in the fish fauna of a small lake, Lake Opinicon, Ontario. J. Fish. Res. Bd. Can. 23 (12): 1845-1873.

Lagler, K., J. Bardach, R. Miller \& D. Passino, 1977. Ichtyology. J. Wiley (ed.). New York. 505 pp.

LAUR, D.R. \& A.W. Ebeling, 1983. Predator-prey relationships in surfperches. Env. Biol. Fish. 8 (314): 217-229.

LIEM, K.F., 1967. A morphological study of Luciocephalus pulcher, with notes on gular elements in other recent teleosts. J. Morph. 121: 103-134.

LIEM, K.F., 1978. Modulatory multiplicity in the functional repertoire of the feeding mechanism in cichlid fishes. I Piscivores. J. Morph. 158: 323-360.

Magnan, P. \& G.J. FitzGerald, 1982. Resource partitioning between brook trout (Salvelinus fontinalis Mitchell) and creek chub (Semotilus atromaculatus Mitchell) in selected oligotrophic lakes of southern Quebec. Can. J. Zool. 60: 1612-1617. 
Margalef, R., 1974. Ecologia. Ed. Omega. Barcelona. 951 pp. Margalef, R., 1983. Limnología. Ed. Omega. Barcelona. $1010 \mathrm{pp}$.

Margalef, R., D. Planas, J. Armengol, A. Vidal, N. Prat, A. Guiset, J. Toja \& M. Estrada, 1976. Limnolo. gía de los embalses españoles. Dir. Gral. de Obras Hidráulicas. MOP. Madrid. 450 pp.

MATTHES, H., 1963. A comparative study of the feeding mechanisms of some african Cyprinidae (Pisces, Cypriniformes). Bijdr. Dierk. 33: 1-36.

Matthwes, W.J., 1982. Small fish community structure in Ozark streams: structured assembly patterns or random abundance of species? Amer. Midl. Nat. 107 (1): 43-54.

Mc Commas, S.R. \& R.W. Drenner, 1982. Species replacement in a reservoir fish community: Silverside feeding mechanics and competition. Can. J. Fish. Aqu. Sci. 39 (6): 815-821.

MoyLe, P.B., 1973. Ecological segregation among three species of minnows (Cyprinidae) in a Minnesota lake. Trans. Amer. Fish. Soc. 4: 795-805.

Muller, M., J.W.M. Osse \& J.H.G. Verhagen, 1982. A quantitative hydrodynamical model of suction feeding in fish. J. Theor. Biol. 95: 49-79.

Muller, M. \& J.W.M. Osse, 1984. Hydrodynamics of suction feeding in fish. Trans. Zool. Soc. Lond. 37: 51-135.

Nilsson, N.A., 1979. Food and habitat of the fish community of the offshore region of Lake Vanern, Sweden. Inst. Freshw. Res. 58: 126-139.

Pitcher, T.J. \& P.J.B. HART, 1982. Fisheries ecology. Ed. Croom Helm. Connecticut. pp 414.

Ringler, N.H., 1979. Prey selection by benthic feeder. From: Stroud and Clepper (eds.), Predatory-pery systems in fishery management: 219-229. Proc. Int. Sym. on predatory-prey systems in fish communities and their role in fisheries management.

Rojo, A. \& P. Ramos, 1982. Contribución al conocimiento de la biometría y osteología de Barbus barbus bocagei, Steindachner, 1866 (Pisces: Cyprinidae). Doñana Acta Vertebrata 9: 27-39.

SCHMITT, R.J. \& J.A. COYER, 1983. Variation in surfperch diets between allopatry and sympatry: circumstantial evidence for competition. Oecologia 58: 402-410.
Schoener, T.W., 1974. Resource partitioning in ecological communities. Science 185: 27-39.

Sokal, R.R. \& F.J. Rohlf, 1979. Biometría. Principios y métodos estadísticos en la investigación biológica. Ed. Blume. Madrid. 832 pp.

STENSON, J.A., 1979. Predator-prey relations between fish and invertebrate prey in some forest lakes. Invest. Freshw. Reserv. 58: 166-183.

STICKNEY, R., 1976. Food habits of georgias estuarine fishes. II Symplurus plaginsa (Pleuronectiformes, Cynoglossidae). Trans. Amer. Fish. Soc. 105 (2): 202-207.

Unger, P.A. \& Jr. W.M. LewIS, 1983. Selective predation with respect to body size in a population of the fish Xelomelaniris venezuelae (Atherinidae). Ecology 64 (5): 1136-1144.

URIBE-ZAmora, M., 1975. Selection des proies par le filtre branchial de la carpe miroir (Cyprinus carpio L.). Tesis Doctoral. Universidad Claude Bernard. Lyon. pp 127.

VAN HASSElT, M.J.F.M., 1979. Relationship between move. ment and structure in the jaw apparatus of some Labrinae (Pisces, Perciformes). A deductive, functional-morphological approach. Doctoral Thesis. University of Leiden. Holand. pp. 177.

VAN LeEwen, J.L. \& M. Muller, 1984. Optimun sucking techniques for predatory fish. Trans. Zool. Soc. Lond. 37: 137-169.

VAn OIJEN, M.J.P., 1982. Ecological diferentiation among the piscivorous haplochromine cichlids of Lake Victoria (East Africa) Neth. Zool. 32 (3): 336-363.

Walsh, G. \& G.J. FitzGERALD, 1984. Resource utilization and coexistence of three species of sticklebacks (Gasterosteidae) in tidal salt-marsh pools. J. Fish. Biol. 25: 405-420.

WERNER, E.E., 1977. Species packing and niche complementarity in three sunfishes. Amer. Natur. 111 (979): 553-578.

WERnER, E.E., 1979. Niche partitioning by food size in fish communities. From: Stroud and Clepper (eds.), Predatorprey systems in fisheries management: $311-322$. Sport fishing institute, Washington.

Werner, E.E. \& D.J. Hall, 1977. Competition and habitat shift in two sunfishes (Centrarchidae). Ecology 58: 869-876. 